\title{
Astrometry of Galactic star-forming regions with VERA
}

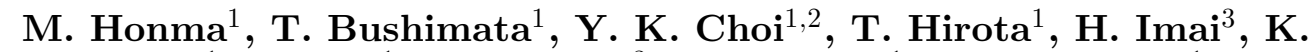
Iwadate $^{1}$, T. Jike ${ }^{1}$, S. Kameno ${ }^{3}$, O. Kameya ${ }^{1}$, R. Kamohara ${ }^{1}$, Y. Kan-ya $^{4}$, N. Kawaguchi ${ }^{1}$, M. Kijima ${ }^{1}$, M. K. Kim ${ }^{1}, 2$, H. Kobayashi ${ }^{1}$, S. Kuji ${ }^{1}$, T. Kurayama ${ }^{1}$, S. Manabe ${ }^{1}$, N. Matsumoto ${ }^{3}$, T. Miyaji ${ }^{1}$, T. Nagayama $^{3}$, A. Nakagawa ${ }^{3}$, K. Nakamura ${ }^{3}$, C.-S. Oh ${ }^{1}$, T. Omodaka ${ }^{3}$, T. Oyama ${ }^{1}$, S. Sakai ${ }^{1}$, K. Sato ${ }^{1}$, M. Sato ${ }^{1}, 2$, T. Sasao ${ }^{5}$, K. M. Shibata $^{1}$, Y. Tamura ${ }^{1}$, and K. Yamashita ${ }^{1}$

${ }^{1}$ Mizusawa VERA observatory, NAOJ ${ }^{2}$ University of Tokyo, ${ }^{3}$ Kagoshima University,

${ }^{4}$ Yonsei University, ${ }^{5}$ Ajou University,

email: mareki.honma@nao.ac.jp

\begin{abstract}
We present an overview of recent astrometric results with VERA. Since 2004, we have been conducting astrometry of tens of Galactic maser sources with VERA, and recently obtained trigonometric parallaxes for several sources, with distances ranging from $180 \mathrm{pc}$ to 5.3 kpc. In this paper, we briefly summarize the results for Galactic star-forming regions, including S269, Orion-KL, NGC 1333, $\rho$-oph, NGC 281 and others.
\end{abstract}

Keywords. astrometry, Galaxy: disk, stars: formation

\section{VERA project}

VERA (VLBI Exploration of Radio Astrometry) is a new VLBI array dedicated to phase referencing VLBI astrometry. This array consists of four 20-m radio telescopes spread over Japan, with a maximum baseline length of $2300 \mathrm{~km}$. VERA's 20m telescopes are operated in the dual beam regime, so that one can simultaneously observe a reference and a target source to cancel out the phase fluctuations caused by tropospheric variations. VERA's main goal is to explore the 3-D structure and dynamics of the Galaxy based on astrometry of 1000 of Galactic maser sources (for more details on the VERA project, see Kobayashi et al. 2008).

\section{Overview on recent results}

Since 2004 we have been conducting astrometric observations of tens of maser sources, and recently we have succeeded in measuring trigonometric parallaxes and proper motions of some maser sources. One of the highlights is a parallax measurement of the star-forming region S269 (Honma et al. .2007). The parallax of S269 was measured to be $\pi=189 \pm 8$ $\mu$ as $(\mathrm{D}=5.28 \pm 0.24 \mathrm{kpc})$, providing one of the largest distances measured by means of trigonometric parallax. Proper motions of S269 $\mathrm{H}_{2} \mathrm{O}$ masers were used to constrain the Galactic rotation velocity at the position of S269 (located at $13 \mathrm{kpc}$ from the Galaxy center), demonstrating that the rotation curve of the Galaxy is basically flat out to 13 kpc (Honma et al. .2007).

The other major result recently obtained with VERA is the distance measurement of Orion-KL, which is one of the most important star-forming regions in the Galaxy. Hirota et al. (2007) determined the parallax of Orion- $\mathrm{KL} \mathrm{H}_{2} \mathrm{O}$ maser as $\pi=2.29 \pm 0.1$ mas 
$(\mathrm{D}=437 \pm 19 \mathrm{pc})$. This value is slightly smaller than previous estimates of $\sim 480 \mathrm{pc}$, but still is in good agreement with recent measurements using VLBA (Sandstrom et al. 2007; Menten et al. 2007). The distance of the $\rho$-Oph cloud was also measured with VERA by Imai et al.(2007), providing the distance of $178_{-37}^{+18} \mathrm{pc}$. This result is consistent with the results obtained by Loinard et al. (2008) based on VLBA parallax measurements of radio-emitting young stars in this region. For other nearby star-forming regions, Hirota et al.(2008) obtained the parallax distance of NGC 1333 to be $\mathrm{D}=235 \pm 18 \mathrm{pc}$.

Another interesting results from VERA is the absolute proper motion measurements for NGC 281 (Sato et al. 2007). This star-forming region is associated with an HI "superbubble", being located $\sim 300$ pc away from the Galactic plan. Sato et al.(2007) found that NGC 281 system is moving away from the Galactic plane at the velocity of $\sim 20 \mathrm{~km} / \mathrm{s}$, which strongly supports an idea that a super-bubble was formed by a blow-out from the Galactic plane, most likely by supernovae.

In addition to star-forming regions, VERA also observed masers in late-type stars. Choi et al.(2008) recently succeeded in astrometry of a super giant star VY CMa, providing the parallax distance of $1.1 \pm 0.1 \mathrm{kpc}$. They also matched the $\mathrm{H}_{2} \mathrm{O}$ maser map with the $\mathrm{SiO}$ maser map and determined a position in the $\mathrm{H}_{2} \mathrm{O}$ maser map, from which maser spots show rapid expansion away from the central star, explained by the mass loss in this star. Similarly, the study of a semi-regular variable S Crt (Nakagawa et al. 2008) provides the parallactic distance of $430 \pm 25 \mathrm{pc}$.

Summarizing the current status, so far we have detected parallaxes for 7 sources, with distances ranging from $180 \mathrm{pc}$ to $5.3 \mathrm{kpc}$. We are going to observe $70-80$ maser sources every year, and in the next $12-15$ years VERA will observe $\sim 1000$ Galactic maser sources to precisely locate them in the Galaxy's disk. Similar astrometric studies of methanol maser sources are also being conducted with VLBA (Reid 2008), and finally Gaia (Lindegren et al. 2008) is also going to conduct kpc-scale astrometry by observing billions of stars in the Galaxy. Thus by 2020, when the results of all these projects become available, our knowledge on the Galaxy structure will be revolutionized with the help of high-precision astrometry both in radio and optical wavelengths.

\section{References}

Choi, Y. K., et al., 2008, in this volume P.192

Hirota, T., et al., 2007, PASJ, 59, 897

Hirota, M., et al., 2008, PASJ, 60, 37

Honma, M., et al., 2007, PASJ, 59, 889

Imai, H., et al., 2007, PASJ, 59, 1107

Kobayashi, H., et al., 2008, in this volume p.148

Lindegren, L., et al., 2008, in this volume p.217

Loinard, L., et al., 2008, in this volume p.186

Menten, K., et al., 2007, A\&\&A, 474, 515

Nakagawa, A., et al., 2008, in this volume p.206

Reid, M. 2008, in this volume p.141

Sato, M., et al., 2007, PASJ, 59, 743

Sandstrom, M., et al., 2007, ApJ, 667, 1161 